\title{
Unexpected effects of peripherally administered kynurenic acid on cortical spreading depression and related blood-brain barrier permeability
}

This article was published in the following Dove Press journal:

Drug Design, Development and Therapy

13 September 2013

Number of times this article has been viewed

\section{Gáspár Oláh' \\ Judit Herédi' \\ Ákos Menyhárt' \\ Zsolt Czinege ${ }^{2}$ \\ Dávid Nagy' \\ János Fuzik' \\ Kitti Kocsis' \\ Levente Knapp' \\ Erika Krucsó' \\ Levente Gellért ${ }^{\prime}$ \\ Zsolt Kis \\ Tamás Farkas' \\ Ferenc Fülöp ${ }^{3}$ \\ Árpád Párdutz ${ }^{4}$ \\ János Tajti ${ }^{4}$ \\ László Vécsei ${ }^{4}$ \\ József Toldi'}

'Department of Physiology, Anatomy and Neuroscience, ${ }^{2}$ Department of Software Engineering, ${ }^{3}$ Institute of Pharmaceutical Chemistry and MTA-SZTE Research Group for Stereochemistry, ${ }^{4}$ Department of Neurology and MTA-SZTE

Neuroscience Research Group,

University of Szeged, Szeged, Hungary

Correspondence: József Toldi

Department of Physiology, Anatomy and Neuroscience, University of Szeged, Közép fasor 52, H-6726, Szeged, Hungary

Tel +3662544153

Fax +36 6354 429l

Email toldi@bio.u-szeged.hu
Abstract: Cortical spreading depression (CSD) involves a slowly-propagating depolarization wave in the cortex, which can appear in numerous pathophysiological conditions, such as migraine with aura, stroke, and traumatic brain injury. Neurons and glial cells are also depolarized transiently during the phenomena. CSD is followed by a massive increase in glutamate release and by changes in the brain microcirculation. The aim of this study was to investigate the effects of two $\mathrm{N}$-methyl-D-aspartate receptor antagonists, endogenous kynurenic acid (KYNA) and dizocilpine, on CSD and the related blood-brain barrier (BBB) permeability in rats. In intact animals, KYNA hardly crosses the BBB but has some positive features as compared with its precursor L-Kynurenine, which is frequently used in animal studies (KYNA cannot be metabolized to excitotoxic agents such as 3-hydroxy-L-kynurenine and quinolinic acid). We therefore investigated the possible effects of peripherally administered KYNA. Repetitive CSD waves were elicited by the application of $1 \mathrm{M} \mathrm{KCl}$ solution to the cortex. Direct current-electrocorticograms were measured for 1 hour. Four parameters of the waves were compared. Evans blue dye and fluorescent microscopy were used to study the possible changes in the permeability of the BBB. The results demonstrated that $N$-methyl-D-aspartate receptor antagonists can reduce the number of CSD waves and decrease the permeability of the BBB during CSD. These results suggest that KYNA itself or its derivatives may offer a new approach in the therapy of migraines.

Keywords: blood-brain barrier, cortical spreading depression, glutamate receptors, kynurenic acid, kynurenines, NMDAR

\section{Introduction}

Cortical spreading depression (CSD), discovered by Leao in 1944, is a slowlypropagating, transient, negative direct current (DC) shift accompanied by depressed neuronal bioelectrical activity. The main event during CSD is the depolarization of a critical mass of brain tissue and a massive elevation in glutamate release associated with an increase in extracellular potassium ion concentration, water influx into the cells, and shrinkage of the extracellular space ${ }^{1-4}$ There are also changes in immediate early genes, neurotransmitters, growth factors, etc. ${ }^{5}$

Some years ago, it was observed that a high glutamate level reduced the function of the blood-brain barrier (BBB) via endothelium-expressed $N$-methyl-D-aspartate receptor (NMDAR)-dependent occludin phosphorylation. ${ }^{6}$ Moreover, CSD initiates disruption of the BBB via a matrix metalloprotease-dependent mechanism. ${ }^{7}$

Despite intensive research, the exact pathomechanism of migraine and its connection with CSD are not completely understood. Various results have indicated that CSD is associated with changes in the diameter of blood vessels in the cortical 
surface, ${ }^{8}$ while other authors have described it as a phenomenon following acute cortical injury, including brain ischemia, ${ }^{9}$ and yet others consider it to be an electrophysiological substrate of migraine aura, which may induce headache. ${ }^{10,11}$

In recent years, we have studied the effects of L-Kynurenine (KYN) and some derivatives of kynurenic acid (KYNA) on different animal models of migraine. ${ }^{12-14}$ It was found, for instance, that KYN suppressed CSD waves and could mitigate some of the labeling thought to be experimental signs of migraine (eg, c-fos immunoreactivity and neuronal nitric oxide synthase). ${ }^{12-15}$ It is widely accepted that KYN (the precursor of KYNA) can readily cross the BBB, but that KYNA itself, the only known endogenous NMDAR antagonist, hardly crosses the BBB at all, ${ }^{16}$ whereas there is evidence that peripherally administered KYNA can penetrate the intact $\mathrm{BBB} .{ }^{17,18}$ Furthermore, published data indicate that CSD results in a disruption of the BBB. ${ }^{7,19}$ Since NMDARs play a decisive role in the generation and propagation of $\mathrm{CSD},{ }^{3,20}$ and data are accumulating which suggest that endogenous KYNA may act as a modulator at various levels of the pathomechanism of migraine, ${ }^{21}$ it seemed plausible to study the effects on CSD of exogenously administered KYNA.

Although little is yet known about the connections between migraine, CSD, and changes in BBB permeability, such a relationship appears more than probable. Accordingly, we have now studied the $\mathrm{KCl}$-induced $\mathrm{CSD}$ and changes in BBB permeability after the administration of KYNA and, as a positive control, dizocilpine (MK-801; a well-known noncompetitive antagonist of NMDARs) in order to elucidate whether or not these neuroactive molecules act in parallel on CSD and BBB permeability.

\section{Materials and methods Animals}

The National Institutes of Health principles of animal care and the protocol for animal care, approved by both the Hungarian Health Committee (1998) and the European Communities Council Directive (86/609/EEC), were strictly followed. Every effort was made to limit the number of animals and their suffering. Fifteen male Wistar rats (270 $\pm 21 \mathrm{~g})$ (Charles River, Wilmington, MA, USA) were used for the electrophysiological portion of this study, and an additional 17 for histology. The animals were kept under 12-hour light and 12-hour dark conditions and were raised with free access to water and food pellets. The room temperature was $22^{\circ} \mathrm{C} \pm 1{ }^{\circ} \mathrm{C}$.

\section{Surgery}

All the procedures were carried out under deep nembutal anesthesia, achieved with a dose of $60 \mathrm{mg} / \mathrm{kg}$ Euthasol ${ }^{\circledR}$ (AST Farma, Oudewater, the Netherlands), followed by $20 \mathrm{mg} / \mathrm{kg}$ hourly. A trachea cannula was inserted and the head of the animals was fixed in a stereotaxic frame (David Kopf Instruments, Tujunga, CA, USA). After exposure of the skull, two holes were drilled through the left side of the skull under cooling with saline; one hole facilitated DCelectrocorticogram (DC-ECoG) recording above the primary somatosensory cortex ( $3 \mathrm{~mm}$ caudally; $5 \mathrm{~mm}$ laterally from the bregma) and the other allowed access for the induction of CSD ( $7 \mathrm{~mm}$ caudally and $5 \mathrm{~mm}$ laterally) (Figure 1 ). The dura mater and arachnoidea were carefully removed from the posterior hole.

\section{Electrophysiology}

A glass microelectrode filled with $150 \mathrm{mM}$ saline (resistance 0.8-1.2 M $\Omega$ ) connected to an $\mathrm{Ag} / \mathrm{AgCl}$ wire was inserted into the cortex to a depth of 1000-1200 $\mu \mathrm{m}$, corresponding to cortical layer $\mathrm{V}$. The control group $(\mathrm{n}=5)$ received $1 \mathrm{~mL}$ of phosphate buffer $(0.2 \mathrm{M}, \mathrm{pH}=7.4)$. The KYNA group $(\mathrm{n}=5)$ was treated with $300 \mathrm{mg} / \mathrm{kg} \mathrm{KYNA}$ (Sigma-Aldrich, St Louis, MO, USA) dissolved in $400 \mu \mathrm{L}$ of $1 \mathrm{M} \mathrm{NaOH}$ and $600 \mu \mathrm{L}$ of phosphate buffer. MK-801 (Tocris Bioscience, Bristol, UK) was dissolved in phosphate buffer and administered at $2 \mathrm{mg} / \mathrm{kg}$ to the animals in the positive control group $(\mathrm{n}=5)$.

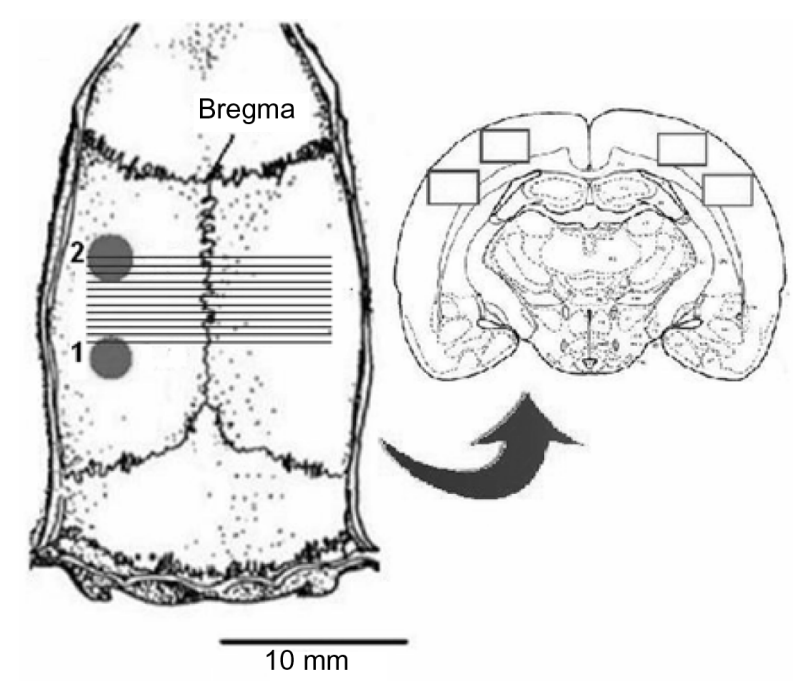

Figure I Explanatory figure relating to the experimental protocol.

Notes: Two holes were drilled through the left side of the skull, one for $\mathrm{KCl}$ application (I) and the other for direct current-electrocorticogram recording (2). The two holes were also drilled in the histological part of this study. The parallel black lines represent the location of the analyzed sections. The rectangles on the right side of the picture show the sites of the digital images captured after Evans blue staining. Abbreviation: $\mathrm{KCl}$, potassium chloride. 
All of these drugs were injected intraperitoneally. The cortex was allowed to recover for 30 minutes following the surgical preparation and treatment. CSD was induced by placing a cotton ball (diameter $1 \mathrm{~mm}$ ) soaked with $1 \mathrm{M} \mathrm{KCl}$ solution on the cortex through the posterior hole. To keep the cotton ball moist, it was impregnated with $5 \mu \mathrm{L}$ of $\mathrm{KCl}$ solution every 15 minutes. The DC-ECoG was measured for 1 hour. Following offline data processing with laboratorydeveloped software, four parameters of the CSD waves were compared between groups: the numbers of CSD episodes per hour, the peak-to-peak amplitude of the waves, the time at half-amplitude, and the area of the waves, calculated via integration. All of these parameters were measured for all waves and then averaged within an animal. The two-sample $t$-test statistical analyses were carried out with Origin 8.1 software (OriginLab, Northampton, MA, USA).

\section{Histology}

For the histological part of the study, the surgical preparation and treatment were the same as described above $(\mathrm{n}=5$ per group). After the 30-minute recovery, $5 \mathrm{~mL} / \mathrm{kg}$ of $2 \%$ Evans blue (EB) dye, dissolved in saline, was administered intravenously at $0.1 \mathrm{~mL} / \mathrm{min}$. CSD waves were evoked by the same method as described above. Two additional animals served as sham-operated controls. The sham preparation was similar to that for experimental animals except that a salineimpregnated cotton ball was applied to the cortex. After 1 hour induction of $\mathrm{CSD}$ by $\mathrm{KCl}$ or saline irrigation, the animals were perfused with cold saline and fixed with $4 \%$ paraformaldehyde dissolved in $0.1 \mathrm{M}$ phosphate buffer. The brains were removed and postfixed overnight in the same paraformaldehyde solution. Sections were cut at a thickness of $20 \mu \mathrm{m}$ with a vibratome (Leica VT 1000S; Leica Microsystems, Wetzlar, Germany). Twelve sections were cut in steps of $500 \mu \mathrm{m}$ from the area of CSD initiation, in the rostral direction. The first section was situated $6 \mathrm{~mm}$ caudally from the bregma. These preparations were mounted on $2 \%$ gelatine-coated slides and covered with Fluoromount (SERVA, Heidelberg, Germany). The slides were examined under a fluorescent microscope (Olympus BX51; Olympus Corporation, Tokyo, Japan) equipped with a DP70 digital imaging system (Olympus Corporation). A Texas red filter (532-587 nm excitation wavelength and $608-683 \mathrm{~nm}$ emission wavelength) was used for the detection of EB fluorescence. Two $1360 \times 1024$ red, green, blue color model pictures were captured from each hemisphere of the sections (Figure 1). After data collection, the pictures were transformed to 8-bit gray-scale images, and the mean intensity was calculated via the following equation:

$$
\bar{I}=\frac{\sum_{i=1}^{n} \sum_{j=1}^{m} I_{i j}}{n \cdot m}
$$

where $I$ is the intensity of pixels, $n$ is the number of pixels in a row and $m$ is the number of pixels in a column. After quantification, the ratio of the intensities of the two hemispheres was calculated and plotted (Figure 2). Repeated-measures one-way analysis of variance (ANOVA) was carried out with Origin 8.1 software.

\section{Results \\ Electrophysiology}

$\mathrm{KCl}$ administration to the cortex resulted in CSD waves with a frequency of $15 \pm 1.1$ /hour (mean \pm standard error of the mean). KYNA suppressed the CSD activity significantly (9.8/hour $\pm 0.9 /$ hour; $P=0.0084$ ), while MK-801 administration completely eliminated the CSD waves (Figure 3A).

Detailed data analysis showed that the application of $1 \mathrm{M}$ $\mathrm{KCl}$ to the cortex in the control group resulted in CSD waves with an amplitude of $21 \mathrm{mV} \pm 0.3 \mathrm{mV}$. However, KYNA $(20.6 \mathrm{mV} \pm 0.7 \mathrm{mV})$ did not cause a significant change in the amplitude of the CSD waves, and no CSD wave was observed following the administration of MK-801 (Figure 3B).

Though KYNA did not induce a significant change in the amplitude of the CSD waves, the time at half-amplitude was significantly shorter after KYNA administration (from 24.9 seconds \pm 0.7 seconds to 21.7 seconds \pm 0.6 seconds; $P=0.0014$ ) (Figure 3C). This is because the CSD waves became sharper as compared with the controls after drug administration (Figure 4).

After integration of the waves, the CSD area in the control group was $48.3 \pm 1.7$. The KYNA treatment produced a significant decline, to $40.1 \pm 2.4(P=0.008)$. MK-801 completely eliminated the CSD activity (Figure 3D).

\section{Histology}

There were no detectable differences between the two hemispheres after 1 hour of saline irrigation in the sham-operated control group $(\mathrm{n}=2)$. In all groups, $\mathrm{KCl}$ was administered to the cortex, as detailed above. In the control and the KYNAtreated group, massive plasma extravasation was detected as intense EB fluorescence within $1000 \mu \mathrm{m}$ of the site of $\mathrm{KCl}$ application (Figure 2, 5). The only exception was the MK-801 group, where the level of fluorescence (extravasation) was half that in the control and KYNA groups. However, the intensity of fluorescence decreased with increasing distance from the site of CSD induction. Fluorescence in 


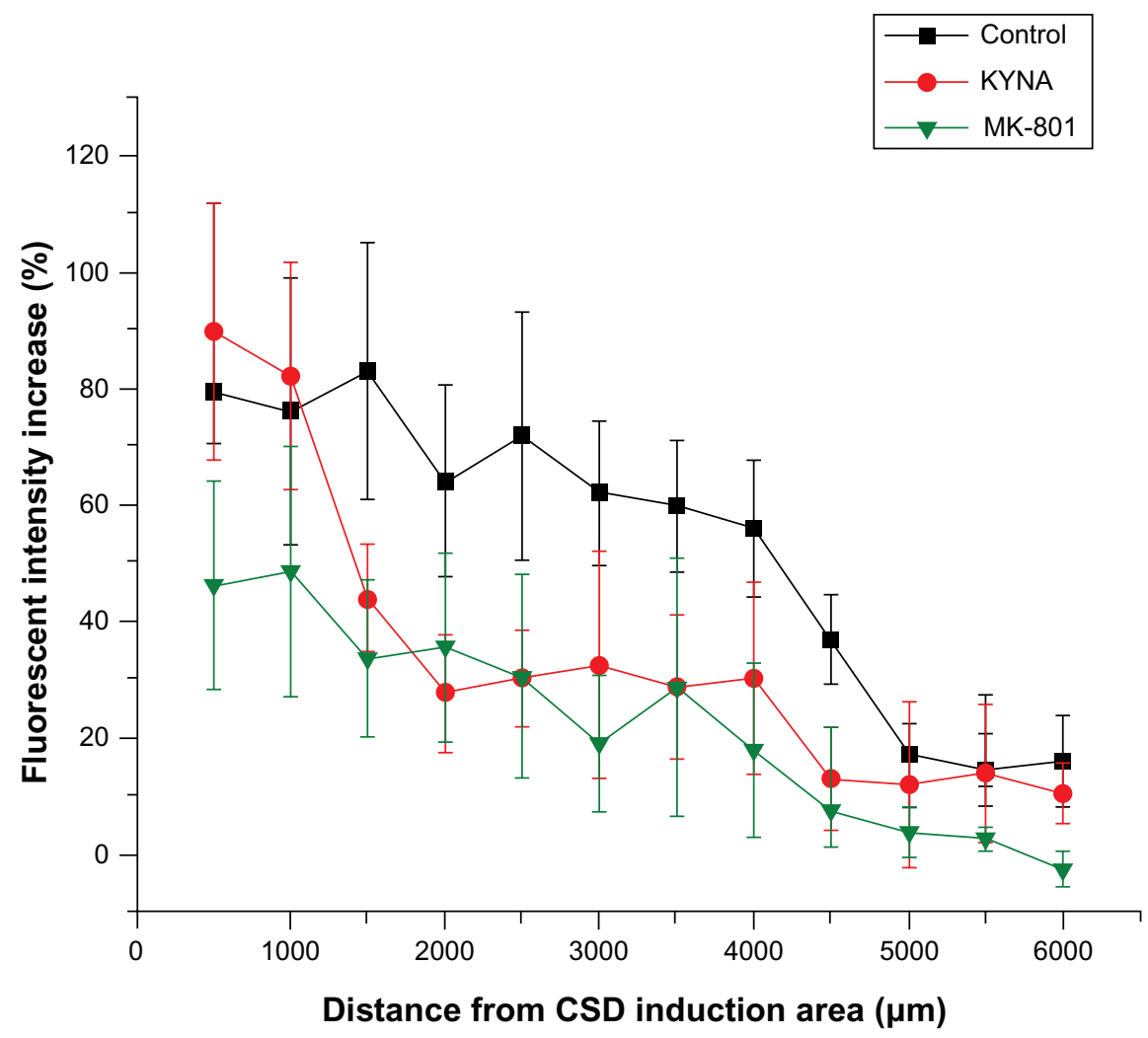

Figure 2 Fluorescence intensity changes caused by plasma extravasation in the three groups.

Notes: Each dot represents a mean intensity ratio ( \pm standard error of the mean) of ten pictures, which were taken from the two hemispheres at the same distance from the $\mathrm{KCl}$ application area. There was no significant difference between the groups according to repeated-measures one way ANOVA.

Abbreviations: ANOVA, analysis of variance; CSD, cortical spreading depression; KYNA, kynurenic acid; MK-80I, dizocilpine; KCl, potassium chloride.

the KYNA-treated animals was only reduced to the levels observed with MK-801 treatment at $\sim 1500 \mu \mathrm{m}$ (Figure 2). Repeated-measures one-way ANOVA did not reveal any significant difference between the groups.

\section{Discussion}

The mechanism of generation of CSD waves, and especially those aspects relating to pathological conditions such as migraine, brain ischemia, and alterations in the $\mathrm{BBB}$, is not yet clear.

A decade ago, it was reported that ischemia and hypoxic cortical depolarization are associated with an intense vasospasm and vasogenic edema. ${ }^{19}$ It was later found that neuronal and glial depolarization and an increase in extracellular glutamate level initiate a cascade that disrupts the BBB in numerous ways. ${ }^{67}$

We have previously investigated the effects of kynurenines on different animal models of migraine. In all of the studied models, KYN (a precursor of KYNA) and other KYNA derivatives proved effective in mitigating the stimulation-induced increase in c-fos immunoreactivity in the caudal trigeminal nucleus ${ }^{12,13}$ or nitroglycerine-induced neuronal nitric oxide synthase. ${ }^{14}$
It was recently described that the administration of KYN significantly decreases the frequency of CSD, an effect due to the increased level of KYNA in the brain..$^{15}$ Although $\mathrm{KYN}$ seems to be neuroprotective in many animal models of migraine and stroke, it sometimes facilitates pathological pathways (our unpublished data). ${ }^{22}$ This may be caused by the metabolization of KYN; it can be converted to excitotoxic 3-hydroxy-L-kynurenine and quinolinic acid in the brain. ${ }^{23}$ Kynurenine aminotransferase converts KYN to KYNA in a unidirectional manner, and peripherally administered KYNA can therefore not be metabolized to excitotoxic agents. ${ }^{24}$ Accordingly, and in light of the fact that CSD increases the permeability of the BBB, it appeared worthwhile to investigate whether systemically administered KYNA influences the CSD activity. In parallel with KYNA, we studied the effects of MK-801 as a positive control.

The aims of this study were to learn whether or not peripherally administered KYNA is able to influence the CSD activity, and whether these neuroactive molecules (which influence the glutamatergic system) act in parallel on CSD and the permeability of the BBB. Our results using these molecules were in accordance with earlier observations and 
A

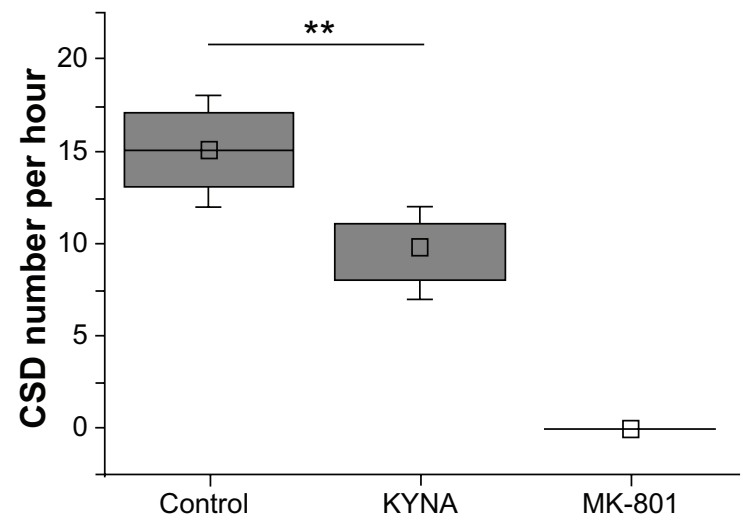

B

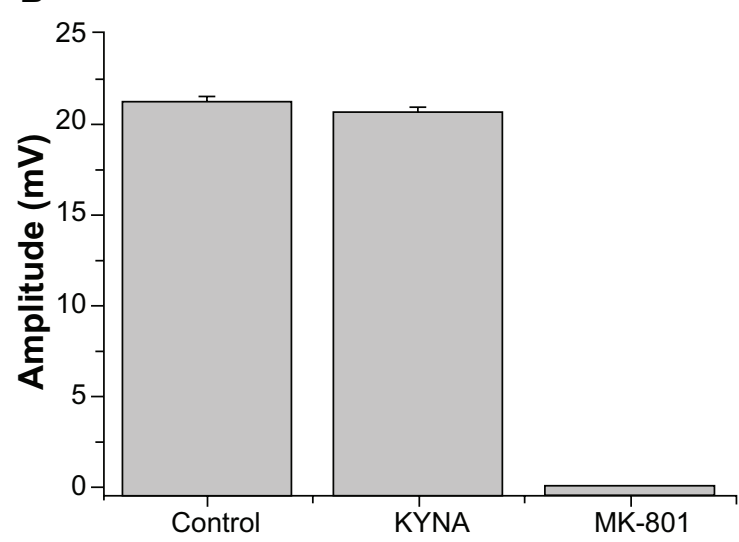

C

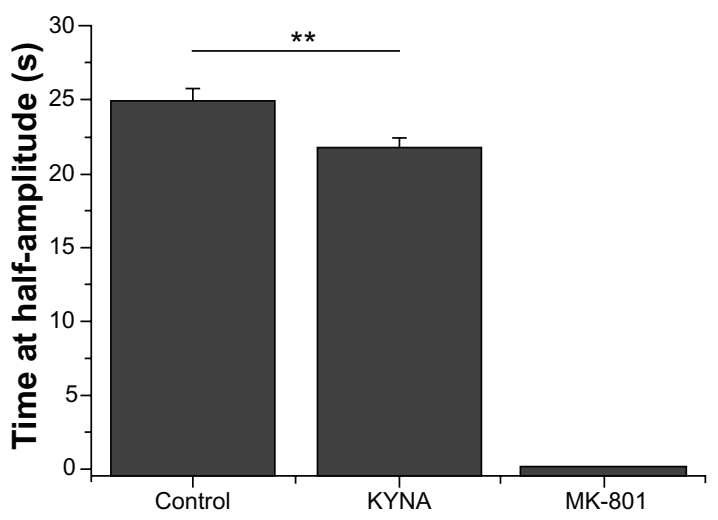

D

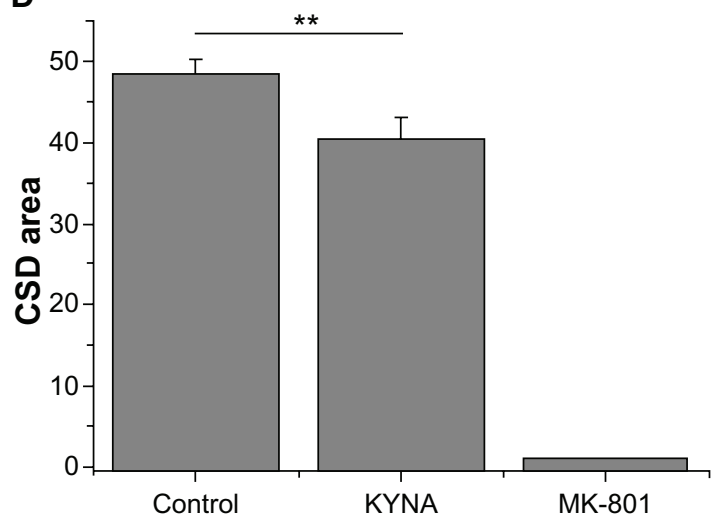

Figure 3 Diagrams of cortical spreading depression (CSD) wave parameters.

Notes: (A) Box diagrams of CSD frequency. The squares inside the boxes indicate the means of the samples. CSD frequency expressed in waves per hour. A significant difference between the control and the kynurenic acid (KYNA)-treated group was indicated by the two-sample t-test. $* * P<0.0 \mathrm{I}$. Dizocilpine (MK-80I) completely blocked the CSD waves. (B) CSD wave amplitudes in $\mathrm{mV}$. No significant difference was detected by two-sample $t$-test. (C) Time at half-amplitude in seconds. Two-sample $t$-test was performed $(* * P<0.01)$ between the control and treated group. (D) Diagram of CSD area. Two-sample $t$-test was performed $(* * P<0.0 \mathrm{I})$ between the control and kynurenic acid (KYNA) groups.

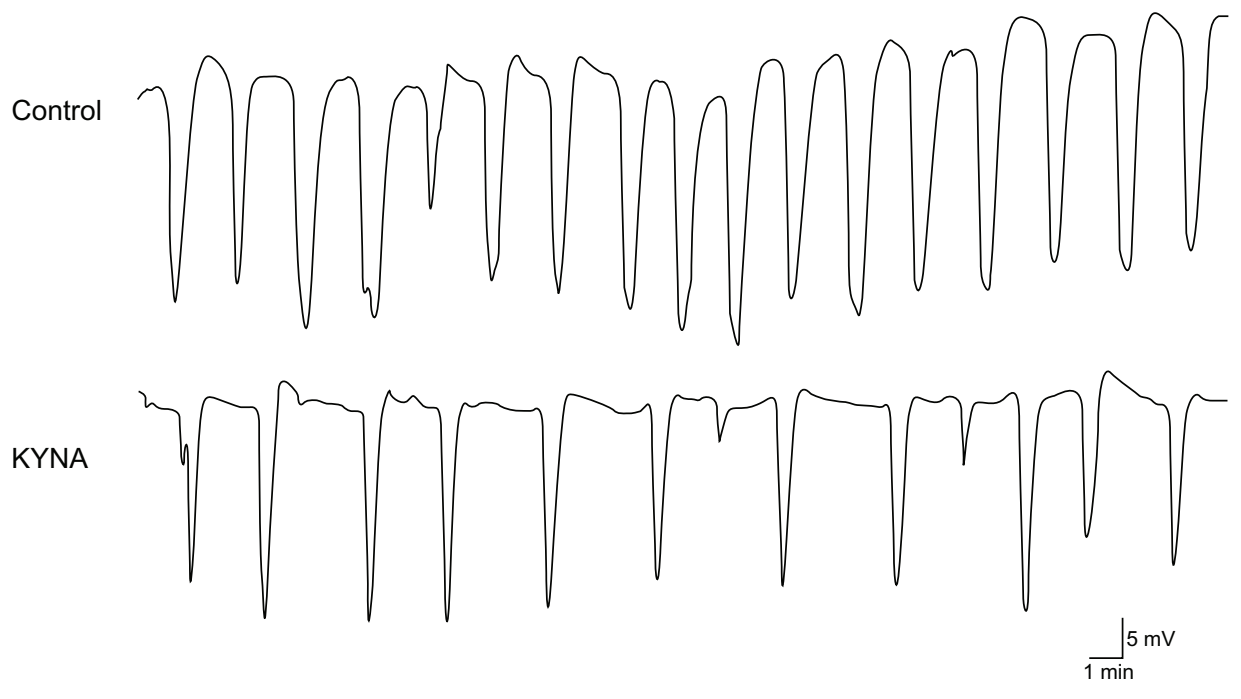

MK-801

Figure 4 Representative cortical spreading depression (CSD) waves recorded in the barrel cortex.

Notes: The uppermost measurement is from a phosphate buffer-treated animal. The CSD wave frequency and the time at half-amplitude were reduced by kynurenic acid (KYNA) treatment, as shown by the second registration. Dizocilpine (MK-80 I) completely blocked the initiation of the waves. Scale bar: I minute; 5 mV. 


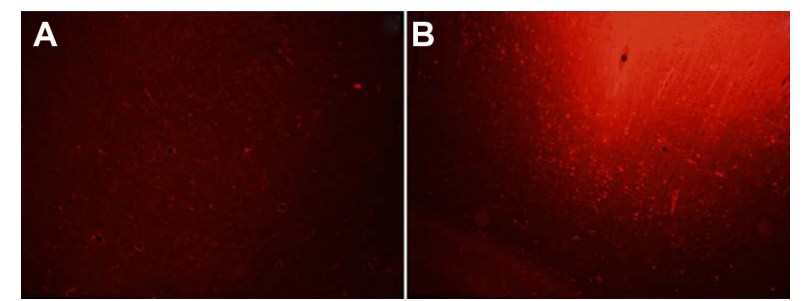

Figure 5 Representative photomicrographs of the cerebral cortex.

Notes: The images were taken from a control animal at $1000 \mu \mathrm{m}$ from the area of cortical spreading depression induction. There was a massive increase in fluorescent intensity on the $\mathrm{KCl}$ treated hemisphere (B) compared to the contralateral cortex (A). Magnification 20x.

Abbreviation: $\mathrm{KCl}$, potassium chloride.

inferences that glutamate is involved in CSD. ${ }^{25,26}$ Results of this study also implicated elements of the pathophysiology of the disorder, including trigeminovascular activation and central sensitization. ${ }^{27}$

The fluorescent dye EB binds to the serum albumin in the blood. Under physiological conditions, albumin cannot pass through the brain capillaries. The intracellular calcium concentration $\left[\mathrm{Ca}^{2+}\right]_{\mathrm{i}}$ demonstrates a laminar difference in the response of the pyramidal neurons to CSD. ${ }^{28}$ Unfortunately, the situation concerning the $\left[\mathrm{Ca}^{2+}\right]_{\mathrm{i}}$ in the endothelial cells is not known, but in general, the linkage between $\left[\mathrm{Ca}^{2+}\right]_{i}$, nitrous oxide, and the integrity of the $\mathrm{BBB}$ is well-based:29,30 elevated $\left[\mathrm{Ca}^{2+}\right]_{i}$ and enhanced neuronal nitric oxide synthase activation lead to robust vasodilatation and protein extravasation, when the albumin-EB complex may appear in the brain tissue following $\mathrm{KCl}$-induced CSD activity.

Although it cannot be excluded that $\mathrm{KCl}$ administration itself may result in increased extravasation, the fact that KYNA and MK-801 administration resulted in reduced or eliminated CSD activity in parallel with decreased extravasation strongly suggests that the waves of cortical depolarization and the level of BBB permeability are related.

CSD waves, the typical electrophysiological signs of cortical depolarizing waves, disappeared completely after MK-801 administration. A similar, but less pronounced effect was observed after KYNA administration. The changes in the electrical signal of the CSD (ie, the decrease in frequency of the DC shifts and the sharpening of the waves) were observed at the beginning of recording, but CSD was elicited only at the beginning of the DC-ECoG measurement. This suggests that peripherally administered KYNA is able to cross the intact $\mathrm{BBB}$ and change the parameters of the waves. In parallel with the electrophysiological results, the administration of these NMDAR antagonists resulted in a gradually declining level of fluorescence, in accordance with increasing distance from the source of the CSD waves (the site of $\mathrm{KCl}$ application).
The reason for this relatively moderate effect of KYNA, as compared with that of MK-801, is probably its increased but limited permeability through the BBB. Under physiological conditions, KYNA is barely able to cross the BBB, ${ }^{16}$ but our results indicate that, in $\mathrm{KCl}$-treated animals, KYNA may pass through the BBB and influence the CSD activity. KYNA presumably mitigates the effects of the glutamate system. KYNA is not only an endogenous NMDAR blocker, but also a noncompetitive inhibitor of the $\alpha$-7-nicotinic acetylcholine receptor, ${ }^{31,32}$ and through this mechanism, KYNA can decrease glutamate release. ${ }^{33}$ In this manner, KYNA can reduce the pathological glutamate level in the cortex during CSD activity and protect the BBB structure. ${ }^{6}$

Our results suggest that, in the course of CSD-induced glutamate release, the permeability of the BBB increases, and systemically administered KYNA can therefore reduce the consequences of CSD.

Recent findings indicated that kynurenines, and particularly KYNA, exhibit a strong modulatory potential on the neuronal structures in the brainstem, which may play a crucial role in the pathogenesis of migraine. ${ }^{34}$ The present data reveal that KYNA may also act in the cortex. These results provide an experimental tool by which to understand the pathomechanism of migraine. However, further studies are needed to clarify the possible role of the kynurenines in the therapy of migraine.

\section{Acknowledgments}

This work was supported by TÁMOP 4.2.2-A-11/KONV2012-0052, OTKA K105077 and TÁMOP 4.2.4. A/2-111-2012-0001 "National Excellence Program - Elaborating and operating an inland student and researcher personal support system".

\section{Disclosure}

The authors report no conflicts of interest in this work.

\section{References}

1. Gardner-Medwin AR. Possible roles of vertebrate neuroglia in potassium dynamics, spreading depression and migraine. J Exp Biol. 1981;95:111-127.

2. Kraig RP, Nicholson C. Extracellular ionic variations during spreading depression. Neuroscience. 1978;3(11):1045-1059.

3. Somjen GG. Mechanisms of spreading depression and hypoxic spreading depression-like depolarization. Physiol Rev. 2001;81(3): 1065-1096.

4. Fabricius M, Jensen LH, Lauritzen M. Microdialysis of interstitial amino acids during spreading depression and anoxic depolarization in rat neocortex. Brain Res. 1993;612(1-2):61-69.

5. Jander S, Schroeter M, Peters O, Witte OW, Stoll G. Cortical spreading depression induces proinflammatory cytokine gene expression in the rat brain. J Cereb Blood Flow Metab. 2001;21(3):218-225. 
6. András IE, Deli MA, Veszelka S, Hayashi K, Hennig B, Toborek M. The NMDA and AMPA/KA receptors are involved in glutamateinduced alterations of occludin expression and phosphorylation in brain endothelial cells. J Cereb Blood Flow Metab. 2007;27(8): 1431-1443.

7. Gursoy-OzdemirY, Qiu J, Matsuoka N, et al. Cortical spreading depression activates and upregulates MMP-9. J Clin Invest. 2004;113(10): 1447-1455.

8. Brennan KC, Beltrán-Parrazal L, López-Valdés HE, Theriot J, Toga AW, Charles AC. Distinct vascular conduction with cortical spreading depression. J Neurophysiol. 2007;97(6):4143-4151.

9. Dohmen C, Sakowitz OW, Fabricius M, et al. Spreading depolarizations occur in human ischemic stroke with high incidence. Ann Neurol. 2008;63(6):720-728.

10. Santos E, Sánchez-Porras R, Dohmen C, Hertle D, Unterberg AW, Sakowitz OW. Spreading depolarizations in a case of migraine-related stroke. Cephalalgia. 2012;32(5):433-436.

11. Zhang X, Levy D, Noseda R, Kainz V, Jakubowski M, Burstein R. Activation of meningeal nociceptors by cortical spreading depression: implications for migraine with aura. J Neurosci. 2010;30(26): 8807-8814.

12. Knyihár-Csillik E, Toldi J, Krisztin-Péva B, et al. Prevention of electrical stimulation-induced increase of $\mathrm{c}$-fos immunoreaction in the caudal trigeminal nucleus by kynurenine combined with probenecid. Neurosci Lett. 2007;418(2):122-126.

13. Knyihár-Csillik E, Toldi J, Mihály A, et al. Kynurenine in combination with probenecid mitigates the stimulation-induced increase of c-fos immunoreactivity of the rat caudal trigeminal nucleus in an experimental migraine model. J Neural Transm. 2007;114(4):417-421.

14. Vámos E, Párdutz A, Varga $H$, et al. 1-kynurenine combined with probenecid and the novel synthetic kynurenic acid derivative attenuate nitroglycerin-induced $\mathrm{nNOS}$ in the rat caudal trigeminal nucleus. Neuropharmacology. 2009;57(4):425-429.

15. Chauvel V, Vamos E, Párdutz A, Vecsei L, Schoenen J, Multon S. Effect of systemic kynurenine on cortical spreading depression and its modulation by sex hormones in rat. Exp Neurol. 2012;236(2):207-214.

16. Fukui S, Schwarcz R, Rapoport SI, Takada Y, Smith QR. Blood-brain barrier transport of kynurenines: implications for brain synthesis and metabolism. J Neurochem. 1991;56(6):2007-2017.

17. Heyes MP, Quearry BJ. Quantification of kynurenic acid in cerebrospinal fluid: effects of systemic and central L-kynurenine administration. J Chromatogr. 1990;530(1):108-115.

18. Scharfman HE, Goodman JH. Effects of central and peripheral administration of kynurenic acid on hippocampal evoked responses in vivo and in vitro. Neuroscience. 1998;86(3):751-764.

19. Koroleva VI, Vinogradova LV. [Ischemic and hypoxic depolarization in the rat neocortex.] Zh Vyssh Nerv Deiat Im I P Pavlova. 2000;50(4): 612-623. Russian [with English abstract].
20. Lauritzen M, Hansen AJ. The effect of glutamate receptor blockade on anoxic depolarization and cortical spreading depression. J Cereb Blood Flow Metab. 1992;12(2):223-229.

21. Fejes A, Párdutz A, Toldi J, Vécsei L. Kynurenine metabolites and migraine: experimental studies and therapeutic perspectives. Curr Neuropharmacol. 2011;9(2):376-387.

22. Roussel S, Pinard E, Seylaz J. Kynurenate does not reduce infarct size after middle cerebral artery occlusion in spontaneously hypertensive rats. Brain Res. 1990;518(1-2):353-355.

23. Schwarcz R, Pellicciari R. Manipulation of brain kynurenines: glial targets, neuronal effects, and clinical opportunities. J Pharmacol Exp Ther. 2002;303(1):1-10.

24. Turski WA, Schwarcz R. On the disposition of intrahippocampally injected kynurenic acid in the rat. Exp Brain Research. 1988;71(3): $563-567$.

25. Longoni M, Ferrarese C. Inflammation and excitotoxicity: role in migraine pathogenesis. Neurol Sci. 2006;27(Suppl 2):S107-S110.

26. Ramadan NM. The link between glutamate and migraine. CNS Spectr. 2003;8(6):446-449.

27. Andreou AP, Goadsby PJ. Therapeutic potential of novel glutamate receptor antagonists in migraine. Expert Opin Investig Drugs. 2009;18(6):789-803.

28. Gniel HM, Martin RL. Changes in membrane potential and the intracellular calcium concentration during CSD and OGD in layer V and layer II/III mouse cortical neurons. J Neurophysiol. 2010;104(6): 3203-3212.

29. Chen Y, McCarron RM, Azzam N, et al. Endothelin-1 and nitric oxide affect human cerebromicrovascular endothelial responses and signal transduction. Acta Neurochir Suppl. 2000;76:131-135.

30. Thiel VE, Audus KL. Nitric oxide and blood-brain barrier integrity. Antioxid Redox Signal. 2001;3(2):273-278.

31. Hilmas C, Pereira EF, Alkondon M, Rassoulpour A, Schwarcz R, Albuquerque EX. The brain metabolite kynurenic acid inhibits alpha7 nicotinic receptor activity and increases non-alpha7 nicotinic receptor expression: physiopathological implications. J Neurosci. 2001;21(19): 7463-7473.

32. Stone TW, Forrest CM, Mackay GM, Stoy N, Darlington LG. Tryptophan, adenosine, neurodegeneration and neuroprotection. Metab Brain Dis. 2007;22(3-4):337-352.

33. Konradsson-Geuken A, Wu HQ, Gash CR, et al. Cortical kynurenic acid bi-directionally modulates prefrontal glutamate levels as assessed by microdialysis and rapid electrochemistry. Neuroscience. 15;169(4): 1848-1859.

34. Tajti J, Szok D, Párdutz A, et al. Where does a migraine attack originate? In the brainstem. $J$ Neural Transm. 2012;119(5):557-568.
Drug Design, Development and Therapy

\section{Publish your work in this journal}

Drug Design, Development and Therapy is an international, peerreviewed open-access journal that spans the spectrum of drug design and development through to clinical applications. Clinical outcomes, patient safety, and programs for the development and effective, safe, and sustained use of medicines are a feature of the journal, which

\section{Dovepress}

has also been accepted for indexing on PubMed Central. The manuscript management system is completely online and includes a very quick and fair peer-review system, which is all easy to use. Visit http://www.dovepress.com/testimonials.php to read real quotes from published authors. 